FOUNDATIONS

ADVANCES

ISSN 2053-2733
Keywords: book review; group theory; graph theory.

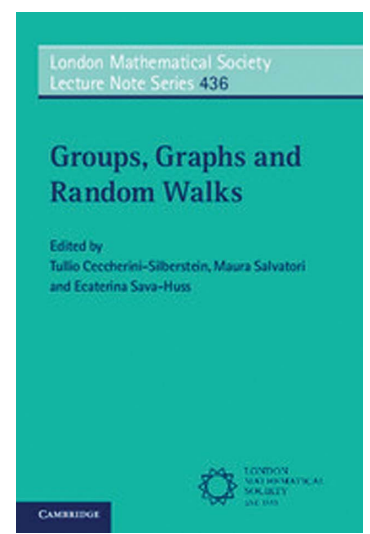

(C) 2018 International Union of Crystallography

\section{Groups, Graphs and Random Walks. Edited by Tullio Ceccherini-Silberstein, Maura Salvatori and Ecaterina Sava- Huss. Cambridge University Press, 2017. Pp. 536. Price GBP 65.00 (paperback). ISBN 9781316604403.}

\author{
Jean-Guillaume Eon*
}

Universidade Federal do Rio de Janeiro, Instituto de Química, Avenida Athos da Silveira Ramos, No. 149, Bloco A - $7^{\circ}$ andar, Centro de Tecnologia, Cidade Universitária, Rio de Janeiro RJ 21941-909, Brazil. *Correspondence e-mail: jgeon@iq.ufrj.br

The book is a collection of original papers written by participants of a conference held in Cortona, Italy, on the occasion of the 60th birthday of Wolfgang Woess, Professor at the Graz University of Technology and currently chair of the Institute of Discrete Mathematics. Most contributors are former students or close collaborators of Wolfgang Woess and present the recent developments of topics that have been of interest to him during his career. All parts are carefully presented, often tending to be self-contained and well documented. Historical developments as well as significant fields of application of the theory are described for the enlightenment of the reader. It is, however, hard to find a straight line of thinking along the successive chapters, even if the different selected themes do show some connectivity. Yet, what is quite natural in a productive life is not necessarily welcome in a book; indeed the outsider cannot see any consistent view of the field emerging from the assortment of texts. Moreover, the book is written by mathematicians and, noticeably, for mathematicians working in the field: this makes it a hard, sometimes impossible, read for non-specialists. Such topics as wreath products, random walks on groups, Poisson boundaries of random walks, buildings, amenability of topological groups or ergodic theory do not really have an immediate appeal for crystallographers, even for mathematical crystallographers. But one might be surprised and suddenly discover a small door opening to the field of crystallography. Following the offered (thorny) path may be rewarding, with time, if one accepts the challenge. This review will point at some possible experiences, reflecting nothing more than the author's own limited knowledge.

The first chapter immediately provides a clear idea of what the reader should expect from the whole book. This chapter is entitled Growth of Groups and Wreath Products. For sure, a clear method of construction of wreath products is given at the beginning of the text and everyone with a minimal background in group theory can grasp the concept. But what are wreath products useful for in materials sciences? The reader might know that symmetry groups of non-rigid molecules can be written as wreath products of permutation groups (Balasubramanian, 1979) as can symmetry groups of noncrystallographic nets (Moreira de Oliveira Jr \& Eon, 2014). After recalling some properties of wreath products, the author introduces the concept of growth of a group and there comes an old friend with a new name. The definition uses the Cayley colour graph $C(G, S)$ of a group $G$ with a set $S$ of generators, a graph whose vertices and edges are in correspondence with the elements of $G$ and of the product $S \times G$, respectively. The growth function $g(R)$ of the group is defined as the number of group elements accessible in $C(G, S)$ by a walk of length inferior to the radius $R$ and starting at the root of the graph. The results are gathered together in the growth series, a formal power series. The chapter discusses the growth function of several kinds of groups that can be constructed as wreath products. Let us recall that many (if not all) uninodal periodic nets can be described as Cayley graphs of some space group. The respective growth function is related to the coordination sequence of the net, and in this case it corresponds to polynomial growth, a result related to the Gromov theorem. But the author is mostly interested in groups of exponential or intermediate growth. 
The small chapter, ch. 6, entitled Amenability of Trees, is a real gem, brilliant and entirely self-contained, though a significant number of errors sometimes impairs the understanding. It extends a theorem proved by Woess on the amenability of infinite trees without leaves. A graph $G$ is said to be amenable if it has isoperimetric number $i(G)=0$, where $i(G)$ is defined as the lower bound for the ratio $|\partial A| /|A|$ of the size of the boundary $\partial A$ of a finite subgraph $A$ to the size of $A$. In order to drop the leafless condition, the authors define (i) a trimming operator, which removes all the leaves of a tree $T$ and (ii) an inessential subtree, which is a finite subgraph that can be removed without disconnecting $T$. The theorem is applied to those genealogic trees built in the Galton-Watson process, showing that trees associated with a probability distribution $\left(p_{i}\right)(i \in N)$ are surely amenable iff $p_{0}+p_{1}>0$.

Ch. 10, entitled Schreier Graphs of Grigorchuk's Group and a Subshift Associated to a Nonprimitive Substitution, has a different flavour. The reader will probably enjoy a sequence of elegant and nearly magical manipulations in the field of dynamical systems. The main objective of the chapter is to elucidate the relation between the spectra of the Laplacian on Schreier graphs associated with self-similar groups and Schrödinger operators with aperiodic order. Clearly originating from the study of properties of quasicrystals, the chapter begins with a presentation of subshifts used as mathematical models of aperiodic order in one dimension. A very special subshift $\left(\Omega_{t}, T\right)$ based on a (nonprimitive) substitution $(\tau)$ on a four-letter alphabet is then introduced and thoroughly analysed. In parallel, the so-called Grigorchuk group $G$, taken as an example of a self-similar group of intermediate growth, is described as an automorphism group of the rooted infinite binary tree. The action of $G$ on the different levels of the binary tree gives rise to Schreier graphs and orbital Schreier graphs whose classes of automorphisms lead to the definition of the dynamical system $(X, G)$. The connection between the two objects is established by introducing a mapping of words from $\Omega_{t}$ to $X$. It is thus shown that the action of $G$ provides a uniquely ergodic dynamical system $\left(\Omega_{t}, G\right)$ admitting $(X, G)$ as a factor. Thanks to this connection, known results concerning Schrödinger operators with aperiodic order can be applied to the weighted Laplacian on Schreier graphs, showing that the respective spectra are Cantor sets of Lebesgue measure zero.

The last part of the book (ch. 16), entitled Topics on Mathematical Crystallography, mainly establishes a connection between the concept of a crystallographic tight frame and that of a topological crystal. Here, the reader is ideally transported back to the realm of classical crystallography with periodic ball-and-stick models of crystal structures in $d$-dimensional Euclidean spaces. The chapter represents a continuation of the book by the same author (Sunada, 2013) but introduces the concept of standard realizations of a topological crystal from a new perspective. Strangely enough, there is no formal definition of a topological crystal. We must rely on the above-mentioned book to learn that a 'topological crystal is an infinite-fold regular covering over a finite graph, whose covering transformation is a free abelian group'. In other words, a topological crystal is a model for an ideal crystal structure with a given finite quotient graph. A 1-tight frame is a set of $N$ vectors in a $d$-dimensional space which shares several of the properties of an orthonormal basis and can be generated by a suitable projection of an orthonormal basis in $\mathrm{N}$-dimensional space. Crystallographic tight frames are 1-tight frames that generate a lattice; they generalize the concept of a root system. Given a finite graph $G$, 1-tight frames can be generated by orthogonal projection of the natural (edge) basis of the respective 1-chain group $C 1$. Because the first homology group $H 1$ (sometimes called the cycle-space) of $G$ is a direct summand of $C 1$, projection along the orthogonal space (the co-cycle space) of the edge basis yields a 1-tight frame. Applying the combinatorial Albanese map provides then a harmonic realization of a maximal abelian covering graph $M$ over $G$. In the general case, a topological crystal is a quotient graph of $M$ by a subgroup $H \leq H 1$. The standard realization of the crystal is obtained as above, but using the projection along the direct sum $H \oplus H 1^{\perp}$. The projection of the edge basis is again a crystallographic 1-tight frame with a periodic lattice that is essentially rational. It is a nice conclusion of this analysis that the representative points of the vertices in the standard realization of the crystal are periodically distributed over the lattice associated with the tight frame.

Having just roamed through four of the 16 chapters of the book, the mathematically inclined reader is invited to find her/ his own way in the remaining chapters.

\section{References}

Balasubramanian, K. (1979). Theor. Chim. Acta, 51, 37-54. Moreira de Oliveira, M. Jr \& Eon, J.-G. (2014). Acta Cryst. A70, 217 228.

Sunada, T. (2013). Topological Crystallography - With a View Towards Discrete Geometric Analysis. Surveys and Tutorials in the Applied Mathematical Sciences, Vol. 6. Tokyo: Springer. 